\title{
PERSISTÊNCIA DE IMAZAQUIN EM LATOSSOLO ROXO CULTIVADO COM SOJA ${ }^{1}$
}

MARIA DO CARMO S. S. NOVO ${ }^{2}$; LUCIANO S. P. CRUZ ${ }^{2}$; JOSÉ CARLOS V. N. A. PEREIRA² ${ }^{2}$ WANDERLEY A. TREMOCOLDI ${ }^{2}$ e TOSHIO IGUE ${ }^{2}$

\section{RESUMO}

No ano agrícola de 1988/89 foi realizado um experimento de campo na Estação Experimental de Ribeirão Preto, do Instituto Ag ronômico, com o objetivo de estudar a persistência de imazaquin aplicado em préemergên cia na cultura da soja cv IAC- 8 , em latossolo roxo, textura argilosa e verificar sua influência no rendimento de grãos. $\mathrm{O}$ experimento foi disposto em blocos ao acaso, com parcelas subdivididas e repetidos quatro vezes. Nas parcelas foram estudadas as doses de imazaquin 0 , 75,150 e $300 \mathrm{~g} / \mathrm{ha}$. As linhas das parcelas foram sorteadas e cada época de amostragem de solo foi retirada de uma das entrelinhas constituindo-se nas sub-parcelas. A atividade residual do imazaquin foi avaliada por meio de bioensaios, sendo usado o pepino (Cucum is sativus L. cv Sprent), como planta-teste. O número de dias que cada dose levaria para não apresentar mais efeito residual no solo foi determinada através de análise de regressão polinomial, estabelecida por dose. Verificou-se que onde haviam sido aplicados 75 e $150 \mathrm{~g} /$ ha de imazaquin, aos 10 dias não havia mais efeito no crescimento da planta-teste. Para a dose de $300 \mathrm{~g} / \mathrm{ha}$ somente aos 22 dias deixou de existir efeito residual. Entretanto, aos 60, 48 e 43 dias após a aplicação do produto, respectivamente para 75,150 e $300 \mathrm{~g} / \mathrm{ha}$, novamen te foi observada redução no crescimento da planta-teste com sintomas típicos nas folhas de pepino. Somente a partir de 89 dias para a dose de $75 \mathrm{~g} / \mathrm{ha}$ e 104 dias para as doses 150 e $300 \mathrm{~g} / \mathrm{h}$ a do imazaquin estimou-se que não seria mais observado atividade residual do produto em latossolo roxo. Não houve efeito de doses de imazaquin no rendimento de grãos de soja.

Palavras chave: Herbicida; bioensaio; atividade residual.

\section{ABSTRACT \\ Imazaquin persistence in Rhodic Eutrudox soil cultivated with soybeans}

In order to study the imazaquin persistence in a Rhodic Eutrudox Soil cultivated with soybean cv IAC-8, a field experiment was carried out at Ribeirão Preto Experimental Station, in 1988/89. A complete split-plot randomized block design with four replication was used. In the main plots were studied the rates of $0,75,150$ and $300 \mathrm{~g}$ ai/ha of imazaquin in a pre-emergence application. The plot rows were sorted and soil samples were taken in the it rows sorted subparcelas. The imazaquin residual activity was evaluated by bioassay using cucumber (Cucumis sativus L. cv Sprent) as test-plant. The duration in days of the residual effect of each dose in the soil was estimated using polinomial regres sion analysis established by the dosage. For 75 and $150 \mathrm{~g}$ ai/h a of imazaquin, after 10 days the testplant did not show any effect in the growth. With

1 Aceito para publicação em 02/10/95 e na forma revista em 01/04/96.

2 Pesquisadores Científicos, Instituto Agronômico, Caixa Postal 28, CEP 13.001 -970, Campinas, SP. 
$300 \mathrm{~g}$ ai/ha, the residual effect ceased only after 22 days. Furthermore, in the 60, 48 and 43 days after imazaquin application for the 75,150 and $300 \mathrm{~g}$ ai/ha, respectively, again growth reduction of the test-plant was observed. Only after the 89 days for the $75 \mathrm{~g} / \mathrm{ha}$ dose and 104 for the 105 and $300 \mathrm{~g}$

\section{INTRODUÇÃO}

O período no qual um herbicida permanece biologicamente ativo no solo tem grande importân cia prática na determin ação de seu desempenho (Hiltbold, 1974). Sua dissipação nas condições de campo é resultado de vários processos combinados, tais como, absorção pelas plantas, volatilização, lixiviação e ads orção no solo, e de processos de transformação, incluindo degradação microbiana, química e fotolítica (Renner et al., 1988a). Esses processos podem ser afetados pelas condições ambientais, pelas propriedades do solo, pelas práticas culturais e de manejo e pelas próprias características do herbicida (Adams, 1973; Cheung \& Lehmann, 1985 e Shea, 1985).

O imazaquin é um herbicida pertencente ao gru po das imidazolinonas, o qual controla amplo espectro de plantas daninhas na cultura da soja. Sua molécula é anfótera, ocorrendo no solo, em função do pH, cinco es pécies químicas distintas (Renner et al., 1988b e Mangel, 1991). $\mathrm{Na}$ faixa de $\mathrm{pH}$ normalmente encontrado nos solos brasileiros (4 a 6), predomina a espécie aniônica.

Mangel (1991) relatou que nas condições americanas, na faixa de $\mathrm{pH}$ de 3 a 6 , diferenças de 0,2 a 0,4 unidades de $\mathrm{pH}$, podem dobrar o co eficiente de ad sorção de imazaquin por aumentar a formação de compostos na forma de $\mathrm{COOH}$. Goetz et al. (1986) observaram que a adsorção do imazaquin é dependente da quantidade de óxidos e hidróxidos de ferro e alumínio presentes. Nos solos do Estado de São Paulo, os quais são bastante intemperizados e onde há predominância de argila do tipo 1:1, a ai/ha of imazaquin, the residual activity of the herbicide were not observed any more in the soil. There was no effect of the imazaquin dosage in the yield of the soybeans.

Key words: Herbicide; bioassay; residual activity.

contribuição da matéria orgânica é mínima. Entretanto pode haver adsorção dess a molécula por óxidos e hidróxidos de ferro e alumínio, especialmente em latossolo roxo que é rico nesses colóides.

Estudos de persistência de doses de imazaquin realizados nos Estados Unidos, em vários tipos de solos e com diferentes modos de aplicação, mostraram que sua atividade residual variou de 120 a 150 dias (Renner et al., 1988a e 1988c; Loux et al., 1989 e Mills \& Witt, 1989). Nas condições do Estado de São Paulo, Matallo et al. (1991) verificaram que em latossolo vermelhoescuro e latossolo variação Una, o período residual do imazaquin aplicado em préemergência foi, respectivamente, de 2 e 6 semanas.

Os objetivos principais deste experimento foram: de terminar o período residual de imazaquin aplicado em pré-emergência em latossolo roxo cultivado com soja e verificar a influência do imazaquin no rendimento de grãos de soja.

\section{MATERIAL E MÉTODOS}

O experimento foi instalado em 29/12/88 na Estação Experimental de Ribeirão Preto, SP, do Instituto Agronômico, em condições usuais de cultivo de soja Glycine $\max ($ L.) Merrill cultivar IAC-8. O solo era um latossolo roxo, eutrófico, A moderado, tex tura argilosa (Oliveira \& Prado, 1983), cuja análise química da camada de $0-10 \mathrm{~cm}$ encontra-se na Tabela 1. 0 solo foi corrigido previamente e adubado de acordo com a recomendação de Mascarenhas (1985). 
TABELA 1. Análise química do solo na camada de 0-10cm em Ribeirão Preto, 1988/89.

\begin{tabular}{|c|c|c|c|c|c|c|c|c|c|}
\hline $\mathbf{P}$ & M.O. & pH & $\mathbf{K}^{+}$ & $\mathrm{Ca2}^{+}$ & $\mathrm{Mg2}^{+}$ & $(\mathrm{H}+\mathrm{Al})$ & $\bar{S}$ & $\bar{T}$ & \multirow{3}{*}{$\begin{array}{c}\mathrm{V} \\
\% \\
51\end{array}$} \\
\hline $\mathrm{ug} / 100 \mathrm{~cm}^{3}$ & $\%$ & $\mathrm{CaCl}_{2}$ & \multicolumn{6}{|c|}{$\mathrm{meq} / 100 \mathrm{~cm}^{3}$} & \\
\hline 41 & 3,6 & 4,9 & 0,29 & 2,9 & 0,9 & 4,0 & 4,1 & 8,1 & \\
\hline
\end{tabular}

$\mathrm{O}$ experimento foi instalado em parcelas subdivididas com os tratamentos das parcelas principais dispostos em blocos ao acaso com quatro repetições. As parcelas eram constituídas de seis linhas de soja com $5 \mathrm{~m}$ de comprimento espaçadas de $0,60 \mathrm{~m}$, onde estudourse o efeito das doses de imazaquin de 0, 75, 150 e 300g/ha. Embora o terreno da área experimental fosse plano, para evitar contaminação foi deixado entre blocos $2,4 \mathrm{~m}$ sem plantio e entre parcelas $2 \mathrm{~m}$. A semeadura foi feita manualmente na densidade de 20 sementes por metro linear.

As sementes de soja do tratamento inoculado foram inoculadas com Bradyrhizobium iaponicum estirpe SMS-463 (=29W) na dose de $8 \mathrm{~g}$ de inoculante turfoso por quilograma de semente e, imediatamente plantadas na densidade de 20 sementes por metro linear. Para evitar contaminação, o tratamento não inoculado foi semeado primeiro.

O he rbicida foi aplicado em préemergência, logo após a semeadura da soja, no período da manhã, com pulverizador costal dotado de barra com dois bicos de jato plano da série 110.02 , com consumo de calda correspondente a $500 \mathrm{~L} / \mathrm{ha}$. As condições climáticas no período do experimento encontram-se na Tabela 2.

TABELA 2. Condições climáticas observadas no dia da instalaçãodo experimento e nos períodos entre e durante as amostragens em Ribeirão Preto, 1988/89.

\begin{tabular}{|c|c|c|c|c|c|c|c|}
\hline \multirow[t]{2}{*}{ AMOSTRA } & \multirow[t]{2}{*}{ DATAS } & \multicolumn{3}{|c|}{$\begin{array}{c}\text { TEMPERATURA } \\
\left({ }^{\circ} \mathrm{C}\right)\end{array}$} & \multicolumn{3}{|c|}{$\begin{array}{c}\text { PRECIPITAÇÃO } \\
(\mathrm{mm})\end{array}$} \\
\hline & & Min. & Med. & Max. & -7 dias & 0 dias & +7 dias \\
\hline $0 \mathrm{dia}$ & $29 / 12 / 88$ & 18,8 & 25,4 & 32,0 & 111,7 & 1,0 & 38,9 \\
\hline 14 dias & $11 / 01 / 89$ & 16,6 & 21,2 & 25,8 & 141,0 & 23,1 & 73,2 \\
\hline 28 dias & $25 / 01 / 89$ & 16,9 & 24,1 & 31,2 & 25,2 & 2,9 & 71,9 \\
\hline 42 dias & $09 / 02 / 89$ & 18,2 & 22,2 & 26,1 & 122,7 & 9,1 & 120,5 \\
\hline 55 dias & $22 / 02 / 89$ & 17,9 & 24,1 & 30,3 & 61,8 & 0,0 & 59,9 \\
\hline 81 dias & $20 / 03 / 89$ & 17,1 & 23,5 & 29,8 & 52,2 & 0,0 & 16,7 \\
\hline 97 dias & $05 / 04 / 89$ & 17,2 & 24,7 & 32,2 & 4,5 & 0,0 & 15,1 \\
\hline
\end{tabular}

As subparcelas eram referentes às diferentes épocas de amostragens e, foram tomadas nas entrelinhas das parcelas previamente sorteadas. As amostragens foram feitas logo após a aplicação do produto e aos 14, 28, 42, 55, 81 e 97 dias após.
A atividade residual do imazaquin no latossolo roxo foi avaliada por meio de bioensaio, sendo usado o método de Santelmann et al. (1971) na forma descrita por Novo et al. (1991). Como planta-teste foi usado o pepino (Cucumis sativus $\mathrm{L}$. cv. Sprent). 
Para o estudo da relação entre o efeito de doses de imazaquin e o crescimento da parte aérea do pepino, transformou-se o peso em porcentagem em relação ao desenvolvimento da planta-teste do tratamento testemunha que foi considerado igual a 100. Para a determinação do número de dias que cada dose levaria para não apresentar mais efeito residual, usou-se os dados empregados para o efeito de dose.

Como o herbicida imazaquin pode ser adsorvido por óxidos e hidróxidos de ferro e alumínio presentes no solo, for am realizadas análises mineralógicas qualitativa das amostras de solo coletadas no local do experimento por difratometria do Raio X. As amostras coletadas foram levadas ao laboratório onde se eliminou a matéria orgânica e o ferro livre. A fração argila foi obtida por centrifugação. A amostra de argila foi dividida em duas partes. A primeira parte foi sa tura da com $\mathrm{Mg}^{2+}$, ir radiada, gl ic ol ad a e novamente irradiada. A outra parte foi saturada com $\mathrm{K}^{+}$, irradiada e depois aquecida a 350 e $550{ }^{\circ} \mathrm{C}$; apó s cada aquecimento a amostra foi irradiada novamente. Para es sas amo stras foi usado tubo de cobre e filtro de níquel no intervalo de 2 a 30 graus 20 (Jacks on, 1969). Os minera is de argila foram reconhecidos de acordo com o método de Dixon \& Weed (1977).

Para a determinação dos óxidos de ferro, amostras de solo foram aquecidas com $\mathrm{NaOH} 5 \mathrm{~N}$ (Norrish \& Taylor, 1961) e, após secagem, submetidas à difração de Raio $X$ com tubo de cobalto e filtro de ferro no intervalo de 20 a 50 graus 20 .

Os dados das variáveis avaliadas foram submetidos à analise de variância usando-se o teste F. O efeito de doses por época foi avaliado através de regressão polinomial e o efeito de época foi analisado us ando-se regres são polinomial estabelecida por dose.

\section{RESULTADOS E DISCUSSÃO}

Na Tabela 3 são apresentados os dados da influência de doses de imazaquin, aplicadas em latossolo roxo, sobre o crescimento da parte aérea da planta-teste. Os dados mostram que houve efeito residual deste produto ao longo do tempo, apresentando um comportamento bifásico. $\mathrm{Na}$ primeira fase, ocorreu diminuição rápida da persistência, até um momento onde não mais havia redução no crescimento da planta-teste. Na segunda fase, novamente aparece u ação residual do imazaquin com a mesma intensidade verificada na primeira fase. Renner et al. (1988a) também observaram para imazaquin uma tendência a degradação bifásica. Entretanto, ao contrário do oc or ri do neste ex perimento, es ses au tore s observaram uma rápida diminuição da persistência nos primeiros 30 dias após a aplicação, seguida de uma dissipação mais lenta durante os 120 dias subsequentes. Hurle \& Walker (1980), citados por Renner et al. (1988a) desenvolveram um modelo mate mático para estudos de degradação de herbicidas. Esse modelo é bicompartimentado. Em um dos compartimentos está o produto prontamente disponível e no ou tro o não disponível. Esses autores sugeriram que somente o produto do compartimento disponível está sujei to à absorção e perdas imediatas. No compartimento não disponível a ação seria muito mais lenta.

A análise de variância mostrou interação significativa entre as doses aplicadas e épocas de amostragem o que justificou o estudo do efeito de ép oca dentro de cada dose (Figura 1). Na decomposição do efeito de época dentro de doses em componentes linear, quad rático e cúbico, o teste $\mathrm{F}$ mostrou ser significativo para todas as doses embora, o ajuste para as doses de 75 e $150 \mathrm{~g} / \mathrm{ha}$ tenham sido baixos. Renner et al. (1988a) também observaram o mes mo comportamento para doses de imazaquin aplicadas em préemergência.

Por meio de equações de re gressão polinomial, es timou-se que quando foram aplicados 75 e 150g/ha de imazaquin, 10 dias após a aplicação não mais havia atividade residual do produto. O mesmo oc or re u para a dose de $300 \mathrm{~g} / \mathrm{h}$ a somente aos 22 dias. Entretanto, aos 60, 48 e 43 dias após a ap lic ação do produto, 
respectivamente para 75,150 e $300 \mathrm{~g} / \mathrm{ha}$, novamente foi observado redução na massa da matéria fresca da parte aérea da planta-teste com sintomas de necrose e encarquilhamento nas folhas. Somente a partir de 89 dias, para a dose de $75 \mathrm{~g} / \mathrm{ha}$ e, $104 \mathrm{di}$ as para 150 e $300 \mathrm{~g} / \mathrm{ha} \mathrm{de}$ imazaquin não mais existia atividade residual do produto neste solo.

TABELA 3. Efeito de doses de imazaquin no crescimento da planta-teste (pepino), nas diferentes épocas de amostragem, coeficientes das equações de regressão polinomial e seus coeficientes de determinação. Ribeirão Preto, 1988/89.

\begin{tabular}{|c|c|c|c|c|c|c|c|}
\hline \multirow{3}{*}{$\begin{array}{c}\text { DOSES } \\
(\mathrm{g} / \mathrm{ha})\end{array}$} & \multicolumn{7}{|c|}{$\begin{array}{l}\text { EPOCAS DE AMOSTRAGEM } \\
\text { (dias após a aplicação) }\end{array}$} \\
\hline & 0 & 14 & 28 & 42 & 55 & 81 & 97 \\
\hline & \multicolumn{7}{|c|}{ matéria fresca da parte aérea ( $\mathrm{g} /$ recipiente) } \\
\hline 0 & 2,11 & 2,58 & 2,54 & 1,97 & 2,29 & 2,32 & 2,07 \\
\hline 75 & 1,59 & $2,00 \mathrm{~b}$ & 3,13 & 2,09 & 2,12 & 1,94 & 2,14 \\
\hline 150 & 1,07 & 1,59 & 3,33 & 1,85 & 1,95 & 1,71 & 2,22 \\
\hline 300 & 0,03 & 1,26 & 2,56 & 1,95 & 1,60 & 1,70 & 2,37 \\
\hline$a^{*}$ & $\overline{2}, 1 \overline{1425}$ & $\overline{2}, \overline{5} \overline{8006} \overline{6}$ & $\overline{2}, \overline{5} \overline{3} 63 \overline{1}$ & - & $2,292 \overline{2} 5$ & $2,3239 \overline{2}$ & $2, \overline{0} \overline{5} 500$ \\
\hline $\mathrm{b}$ & $-0,00697$ & $-0,00883$ & 0,01052 & - & $-0,00231$ & $-0,00612$ & 0,00103 \\
\hline c & - & 0,000015 & $-0,000035$ & - & - & 0,000015 & - \\
\hline $\mathrm{R}^{2} / \mathrm{r}^{2} * *$ & 88,88 & 86,02 & 74,99 & - & 82,82 & 73,90 & 85,58 \\
\hline C.V $(\%)$ & 20,2 & 7,4 & 15,2 & 9,3 & 14,6 & 11,7 & 8,9 \\
\hline
\end{tabular}

* Coeficientes das equaçôes de regressão linear ( $\mathrm{a}$ e b) e quadrática (a, b e c).

** Coeficiente de determinção $(\%)$ das equações de regressão linear $\left(r^{2}\right)$ e quadrática $\left(\mathrm{R}^{2}\right)$.

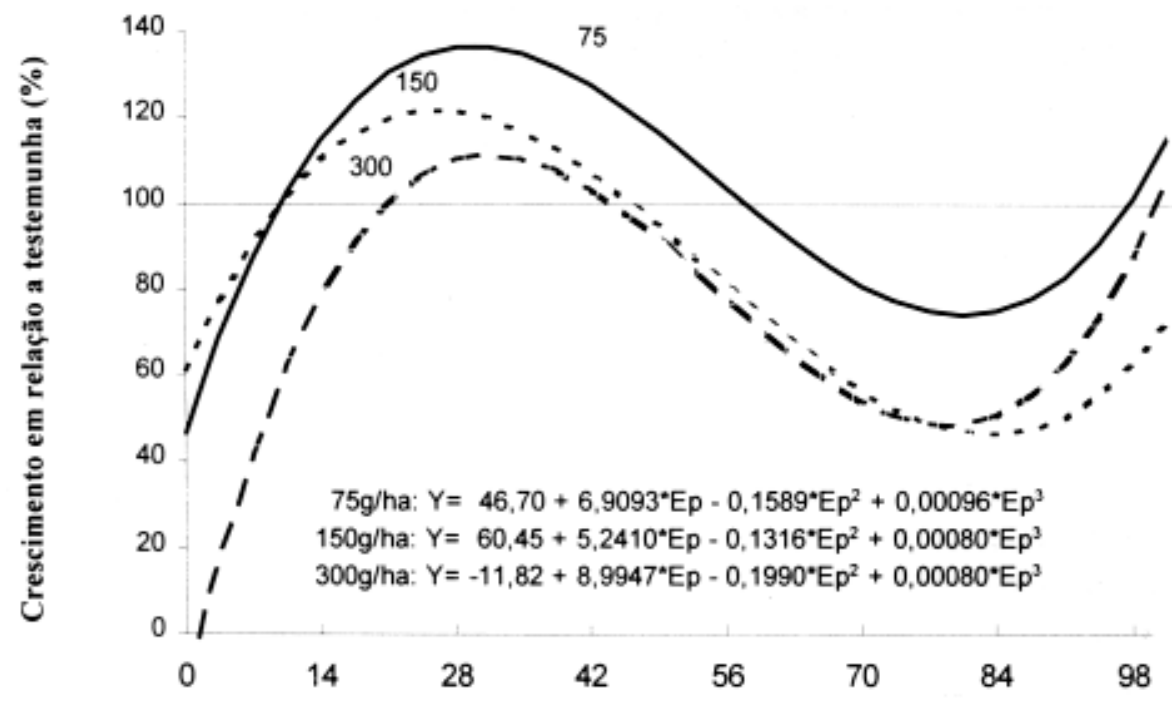

DIAS APÓS A APLICAÇÃo

FIGURA 1. Efeito de doses de imazaquim no crescimento da planta-tes te (pepino) em , relação a testemu nha nas diferentes épocas de amostra gens em Ribeirão Preto, 1988/89. (Ep = em dias). 
A análise mineralógica qualitativa do latossolo roxo mostrou que este solo é constituído essencialmente de caulinita, gibsita e hematita. Em geral, os minerais de argila têm carga negativa porém, a caulinita, em $\mathrm{pH}$ ácido desenvolve cargas positivas em suas arestas (Raij, 1986) mas, sua contribuição ao processo de adsorção aniônica é pequena devido a sua baixa superfície específica.

Por outro lado, os oxihidróxidos de ferro e alumínio (hematita e gibsita) têm ponto de carga zero (PCZ) próximo ao $\mathrm{pH} 7,0$ (Raij, 1986). Isso significa que para $\mathrm{pH}$ inferior a 7,0, que foi $\mathrm{o}$ caso, ocorreu excesso de cargas positivas favorecendo a adsorção iônica do imazaquin, o que foi também demonstrado por Goetz et al. (1986).

Analisando -se o balanço hídrico decendial da Estação Experimental de Ribeirão Preto no período do experimento, determinado pelo método de Thornthwait \& Matter (1955), observou-se relação entre o regime hídrico e a persistência desse produto (Figura 2). Verificouse que até a amostragem realizada aos 81 dias houve excedente hídrico. Entretanto, quando se observou a precipitação acumulada (Figura 3) verificou-se que somente até a amostragem realizada aos 42 dias houve excesso de chuva; a partir dessa amostragem, as chuvas foram mais leves e espaçadas.

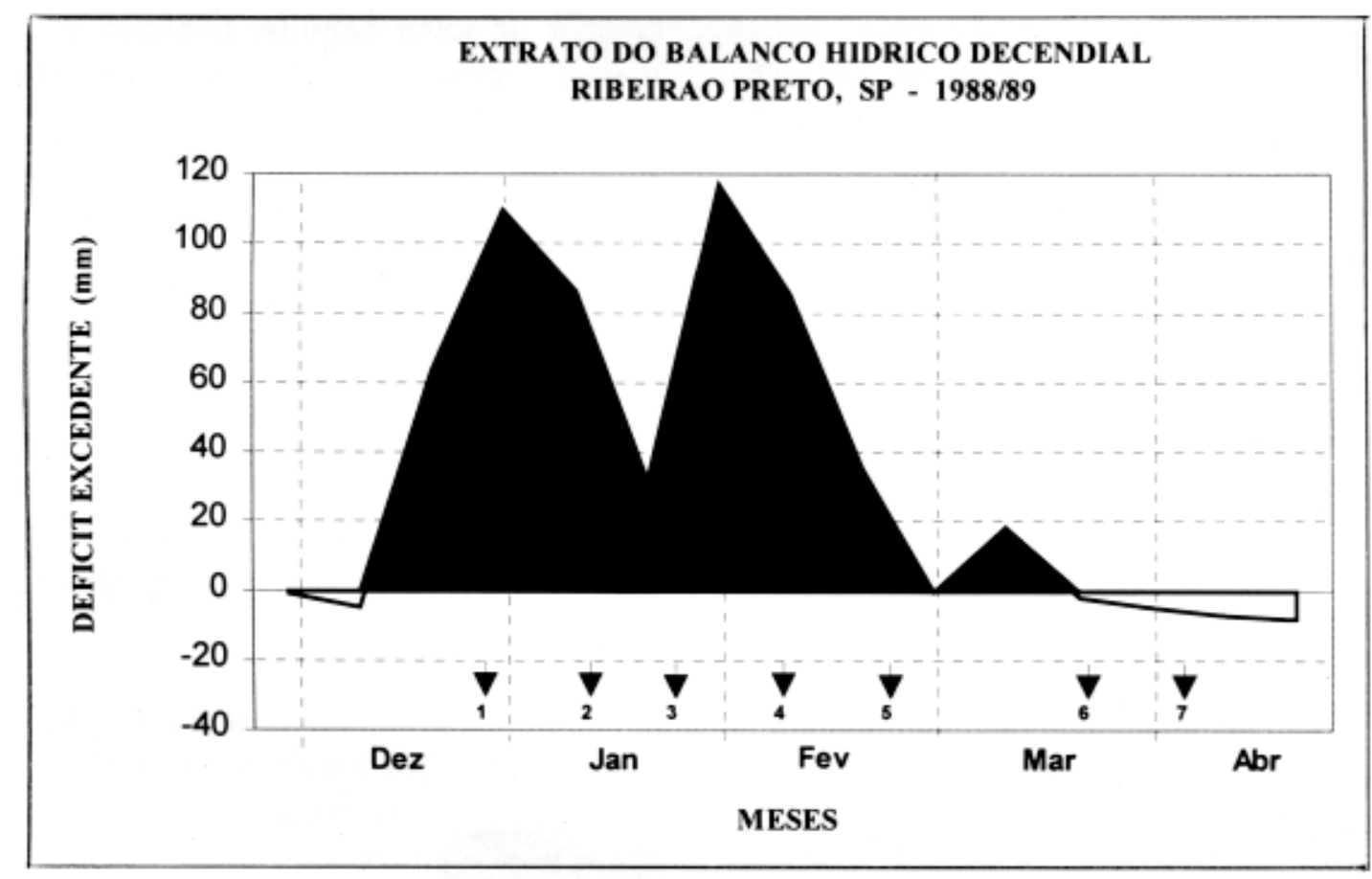

Fonte: Seção de Climatologia Agrícola - IAC

FIGURA 2. Balanço hídrico decendial de dezembro de 1988 a abril de 1989 com as respectivas datas de amostragens (1: 29/12/88; 2: 14/01/89; 3: 25/01/89; 4: 9/02/89; 5: 22/02/89; 6: 20/03/89 e 7 : 05/04/89) em Ribeirão Preto. 


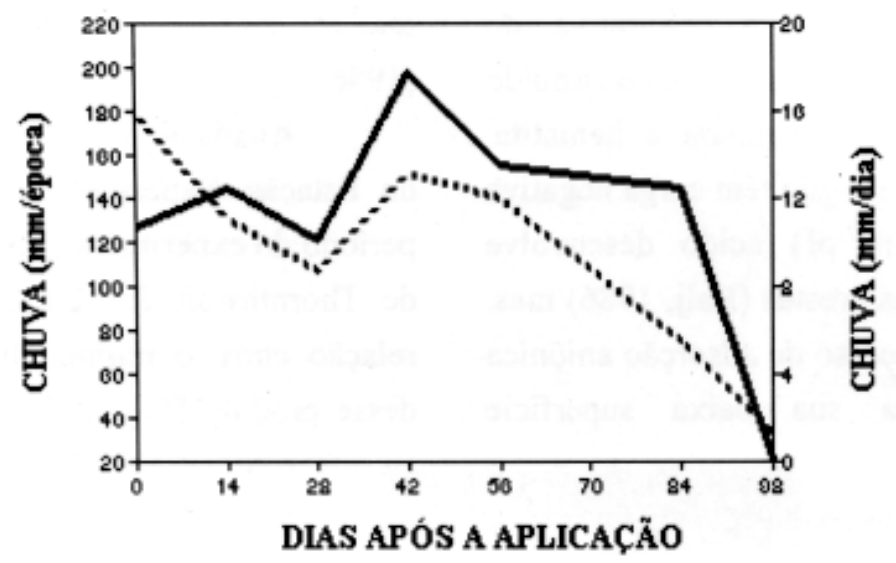

FIGURA 3. Precipitação acumulada por época amostral e por dia em Ribeirão Preto, 1988/89.

Os solos da Estação Experimental de Ribeirão Preto são bastante trabalhados e, como são muito argilosos, sujeitos à compactação. Como o solo recebeu chuva abundante após a aplicação e o imazaquin é fracamente adsorvido pela argila e matéria orgânica (Wolt et al., 1989), presumivelmente, parte do produto que estava na solução do solo deve ter se lixiviado para camadas mais profundas do que a amostrada e parte foi absorvida pela planta. Loux et al. (1989) verificaram que quando chovia logo após a aplicação do imazaquin, ocorria movimentação do herbicida da superfície do solo dentro do perfil, observando-se o fenômeno de perda de superfície. Wolt et al. (1989) relataram que em condições climáticas como as das primeiras amostragen $\mathrm{s}$ deste experimento - solo úmido e temperatura alta houve maior lixiviação. Entretanto, Renner et al. (1988a) verificaram que em qualquer época de amostragem e tanto em aplicações de pré- como de pós-emergência, pouco imazaqu in era detectado abaixo de $10 \mathrm{~cm}$.

Após a amostragem realizada aos 55 dias, verificou-se diminuição bastante acentuada da precipitação mas a te mperatura manteve-se elevada (Tabela 2). Se gundo Mangel (1991) quando a temperatura é elevada, ocorre movimentação ascendente do imazaquin por fluxo capilar devido à evaporação da água. Renner et al. (1988a) verificaram que quando o imazaquin sobe até a superfície do solo por capilaridade, ele fica passível de sofrer hidrólise fotolítica mas esta é bastante limitada, pois a luz não penetra abaixo da superfície do solo.

Goetz et al. (1986) observaram que quan do ocorre secamento temporário do solo e esse volta à capacidade de campo, como as que ocorreram nas amostragens finais, geralmente, aumenta a adsorção do imazaquin mas, esse efeito é mínimo em solo argiloso. O aumento da sorção do imazaquin obtido pela secagem temporária do solo pode ser atribuído à redução na espessura do filme de água que envolve os minerais de argila que servem para concentrar o produto perto da superfície de sorção e/ou para facilitar a sua precipitação.

De acordo com Appleby (1985), a água pode competir por locais de adsorção, deix ando mais herbicida na solução do solo suje ito à absorção e/ou prontamente acessível à degradação e outros modos de dissipação (Basham \& Lavy, 1987).

Não houve resposta às doses de ima zaquin na produtividade de grãos de soja (Tabela 4). 0 mesmo foi obse rvado por Mills \& Witt (1989). 
TABELA 4. Efeito de doses de imazaquin na produtividade de grãos de soja do cultivar IAC-8.

\begin{tabular}{cc}
\hline $\begin{array}{c}\text { DOSES } \\
(\mathrm{g} / \mathbf{h a})\end{array}$ & $\begin{array}{c}\text { Rendimento de grãos } \\
(\mathbf{k g} / \mathbf{h a})\end{array}$ \\
\hline 0 & $2166^{1}$ \\
75 & 2276 \\
150 & 2200 \\
300 & 2401 \\
\hline
\end{tabular}

${ }^{1}$ Médias não significativas ao nivel de $5 \%$ de probabilidade.

A persistência de imazaquin em latossolo roxo cultivado com soja mostrou ser extremamente dependente da umidade do solo. Em lat os solo rox o e com a oc or rên cia de veranicos e chuvas, imazaquin persiste 89 dias, quando da aplicação de $75 \mathrm{~g} / \mathrm{ha}$, e 104 dias quando da aplicação de 150 e 300g/ha.

\section{LITERATURA CITADA}

AD AMS JR, R.S. Fact or s influe ncing soil adsorption and bioactivity of pesticides. Residue Rev., Berlin, v.47, n.1, p.1-54, 1973.

APPLEBY, A.P. Factors in examining fate of herbicides in soil with bioas says. Weed Sci., Champaign, v.33, suppl.2, p.2-6, 1985.

BASHAM, G.W., LAVY, T.L. Microbial and phytolitic dissipation of imazaquin in soil. Weed Sci., Champaign, v.35, n.6, p.865870, 1987.

CHEUNG, H.H., LEHMANN, R.G. Characterization of herbicide degradation under field conditions. Weed Sci., Champaign, v.33, suppl.2, p.7-10, 1985.

DIXON, J.B., WEED, S.B. Mineral in soil environments. Wisconsin: Soil Science Society of America. 1977. 948p.

GOETZ, A.J., WEHTJE, G., WALKER, R.H., HAJEK, B. Soil solution and mobility characterization of imazaquin. Weed Sci., Champaign, v.34, n.5, p.788-793, 1986.

HILTBOLD, A.E. Persistence of pesticides in soil. In: Pesticides in soil and water. Madison: Soil Science Society of America, 1974. p.203-222.

HURLE, K., WALKER, A. Persistence and its prediction. In: Hance, R.J. (ed.) Interactions between herbicides and the soil. New York: Academic Press, 1980. p. 84-122.

JACKS ON, M.L. So il chemical an al ys is. Advanced Course. Madison: Mimeografado. 1969. 895p.

LOUX, M.M., LIEB L, R. A., SLIFE, F.W. Availability and persistence of imazaquin, imazethapyr, and clomazone in soil. Weed Sci., Champaign, v.37, n.2, p.259-267, 1989.

MANGEL, G. Be havi or of imid az olin on es herbicides in soil - A review of the literature. In: SHANER, D.L., O'CONNOR, S.L. (Eds). The imidazolinone herbicides. Boca Raton: CRC Press. 1991. p.191-209 (Capítulo, 16).

MASCAR ENHAS, H.A.A. Soja. In: RAIJ, B. van, SILVA, N.M., BATAGLIA, O.C., QUAGGIO, J.A., HIROCE, R., CANTARELLA, H., CA...... 
JÚNIOR, R., DECHEN, A.R., TRANI, P.E. Recomendações de adubação e calagem para o Estado de São Paulo. Campinas: Instituto Agronômico, 1985. p.20. (Boletim, 100).

MATALLO, M.B., BLANCO, H.G., OLIVEIRA, D.A., TREMOCOLDI, W.A. Persi stência do herbicida imazaquin em dois solos do Estado de São Paulo com soja (Glycine max (L.) Merrill). In: Reunión Argentina sobre la Maleza y su Control, 12. Mar del Plata. Trabajos y Comunicaciones. Asociación Argentina para el Control de Malezas. 1991. p.95-109.

MILLS, J.A., WITT, W.W. Efficacy, phytotoxicity, and persistence of imazaquin, imazethapyr, and clomazone in no-till double-crop soybeans (Glycine max). Weed Sci., Champaign, v.3 7, n.3, p.353-359, 1989.

NORRISH, K., TAYLOR, R.M. The isomorphous replacement of iron by aluminum in soil goethites. Journal of Soil Science, Londres, v.12, n.2, p.294-306, 1961.

NOVO, M.C.S.S., LOPES, E.S., ORTOLAN, M.C.A. Le vant am en to da no du la çã o, persistência de herbicidas e isolamento de Bradyrhizobium sp. de áreas com rotação cana-de-açúcar / Amendoim. Rev. Microbiol., São Paulo, v.22, n.1, p.60-65, 1991.

OLIVEIRA, J.B., PRADO, H. Levantamento pedológico semi-detalhado do Estado de São Paulo: quadrícula de Ribeirão Preto SF-233-V-c-I. Campinas: Instituto Agronômico. 1983. Escala 1: 100.000. (ConvênioEMBRAPA - SAA).
RAIJ, B. van Propriedades ele troquímicas dos solos. In: Simpósio avançado de química e fertilidade do solo. Campinas: Fundação Cargill. 1986. p.9-41.

RENNER, K.A., MEGGITT, W.F., LEAVITT, R.A. Influence of rate, method of application, and tillage on imazaquin persistence in soil. Weed Sci., Champaign, v.36, n.1, p.90-95, 1988a.

RENNER, K.A., MEGGITT, W.F., PENNER, D. Effect of soil $\mathrm{pH}$ on imazaquin and imaze thapyr adsorption to soil and phytotoxicity to corn (Zea mays). Weed Sci., Champaign, v.36, n.1, p.78-83, 1988b.

RENNER, K.A., MEGGITT, W.F., PENNER, D. Response of corn (Zea mays) cultivars to imazaquin. Weed Sci., Champaign, v.36, n.5, p.625-628, $1988 \mathrm{c}$.

SANTELMANN, P.W., WEBER, J.B., WIESE, A.F. A study of soil bioassay technique using prometryne. Weed Sci., Champaign, v.19, n.2, p.170-174, 1971.

SHEA, P.J. Detoxification of herbicide residues in soil. Weed Sci., Champaign, v.33, suppl.2, p.33-41, 1985.

THORNTHWAITE, C.W., MATHER, J.R. The water balance. Centerton, Nova Jersey, Drexel. Institute of Technology, 1955. 104p. (Publications in Climatology, v.8, n.1).

WOLT, J.D., RHODES JR., G.N., GRAVEEL, J.G., GLOSAUER, E.M., AMIN, M.K., CHURCH, P.L. Activity of imazaquin in soil solution as affected by incorporated wheat (Triticum aestivum) straw. Weed Sci., Champaign, v.37, n.2, p. 254-258, 1989. 